\title{
Number of grant applications needed to fund research faculty: a probabilistic analysis
}

\author{
Ashwini R. Sehgal, MD \\ Center for Reducing Health Disparities, Division of Nephrology, Departments of Internal Medicine, Biomedical Ethics, and Epidemiology and \\ Biostatistics, Metro Health Medical Center, Case Western Reserve University, Cleveland, OH, USA.
}

KEY WORDS: grant funding; research faculty; National Institutes of Health

$\mathrm{J}$ Gen Intern Med 33(8):1232-4

DOI: $10.1007 / \mathrm{s} 11606-018-4464-0$

(c) Society of General Internal Medicine 2018

\section{INTRODUCTION}

Obtaining external grant funding is a key milestone for research faculty. Such funds provide resources for conducting research and to meet milestones for tenure and promotion. ${ }^{1}$ Information on the proportion of grant applications funded by the National Institutes of Health (NIH) and other funders is publicly available. For example, the funding rate of NIH research project grants (R01) has decreased from 31 to $20 \%$ over the last 15 years. ${ }^{2}$ Much has been written about how low funding rates create barriers to research success. ${ }^{3}$ However, it is not clear how many grant applications faculty must submit in order to have a high likelihood of being funded.

\section{METHODS}

The binomial distribution was used to determine the number of applications that must be submitted to achieve a desired probability of having at least 1 or 2 applications funded. The probability of having at least 1 application funded is $1-(1-f)^{n}$ where $f$ is the overall proportion of applications funded and $n$ is the number of applications submitted. The probability of having at least 2 applications funded is $1-(1-f)^{n}-n f(1-f)^{n-1}$. For each value of $f$ ranging from 0.05 to 0.50 in increments of 0.05 , the author calculated the smallest $n$ needed to be 10,20 , ..., or $90 \%$ certain of having at least 1 or at least 2 applications funded.

\section{RESULTS}

Table 1 lists the number of grant applications that must be submitted to achieve a desired probability of having at least 1 application funded. Eight applications would need to be submitted to be $80 \%$ certain of having at least 1 application funded if the overall proportion of applications funded is 0.20 . Fourteen applications would need to be submitted to be certain of having at least 2 applications funded (Fig. 1). The

Published online April 30, 2018 number of applications needed decreases as the overall proportion of funding increases.

\section{DISCUSSION}

This analysis indicates that a very large number of grant applications are needed to fund research faculty, especially in the current environment of low funding rates. These findings have important implications for research faculty, department heads, and research policy. Research faculty will need to submit many grant applications to be reasonably certain of receiving some funding. Collaborating with others may be a way to increase the total number of applications. Focusing on funding opportunities with higher funding rates and avoiding opportunities with very low funding rates may also be a useful strategy. ${ }^{3}$ Department heads and academic institutions should avoid basing decisions about research faculty retention or promotion on the results of a handful of grant applications. Other research indicators to consider in evaluating faculty include grant application scores, study section comments, promising pilot data, and research publications. Providing intramural funds of a larger amount and longer duration would also be helpful. Implications for research policy include the following questions. Is it feasible for researchers to submit so many applications in order to be funded? Is writing grant applications taking too much time away from actually conducting research? ${ }^{4}$ Are promising research faculty being let go prematurely by academic institutions? How should the mismatch between the supply of researchers and the availability of research funding be addressed? ${ }^{5}$

Some limitations must be considered in interpreting these findings. The analyses assumed that every application had an independent and average probability of success and that funding follows a binomial distribution. However, it is likely that the quality of the application and preliminary work, whether it is an initial submission or a resubmission, whether the applicant is an experienced or junior investigator, and the availability of support and mentorship at particular institutions will influence the likelihood of funding. These factors could be 
Table 1 Number of Grant Applications That Must Be Submitted to Achieve Desired Probability of Having at Least 1 Application Funded. For Example, 8 Applications Would Need to Be Submitted to Be 80\% Certain of Having at Least 1 Application Funded if the Overall Proportion of Applications Funded Is 0.20

\begin{tabular}{|c|c|c|c|c|c|c|c|c|c|c|}
\hline \multirow[t]{2}{*}{ Probability at least 1 grant funded (\%) } & \multicolumn{10}{|c|}{ Overall proportion of applications funded } \\
\hline & 0.05 & 0.10 & 0.15 & 0.20 & 0.25 & 0.30 & 0.35 & 0.40 & 0.45 & 0.50 \\
\hline 10 & 3 & 1 & 1 & 1 & 1 & 1 & 1 & 1 & 1 & 1 \\
\hline 20 & 5 & 3 & 2 & 1 & 1 & 1 & 1 & 1 & 1 & 1 \\
\hline 30 & 7 & 4 & 3 & 2 & 2 & 1 & 1 & 1 & 1 & 1 \\
\hline 40 & 10 & 5 & 4 & 3 & 2 & 2 & 2 & 1 & 1 & 1 \\
\hline 50 & 14 & 7 & 5 & 4 & 3 & 2 & 2 & 2 & 2 & 1 \\
\hline 60 & 18 & 9 & 6 & 5 & 4 & 3 & 3 & 2 & 2 & 2 \\
\hline 70 & 24 & 12 & 8 & 6 & 5 & 4 & 3 & 3 & 3 & 2 \\
\hline 80 & 32 & 16 & 10 & 8 & 6 & 5 & 4 & 4 & 3 & 3 \\
\hline 90 & 45 & 22 & 15 & 11 & 8 & 7 & 6 & 5 & 4 & 4 \\
\hline
\end{tabular}

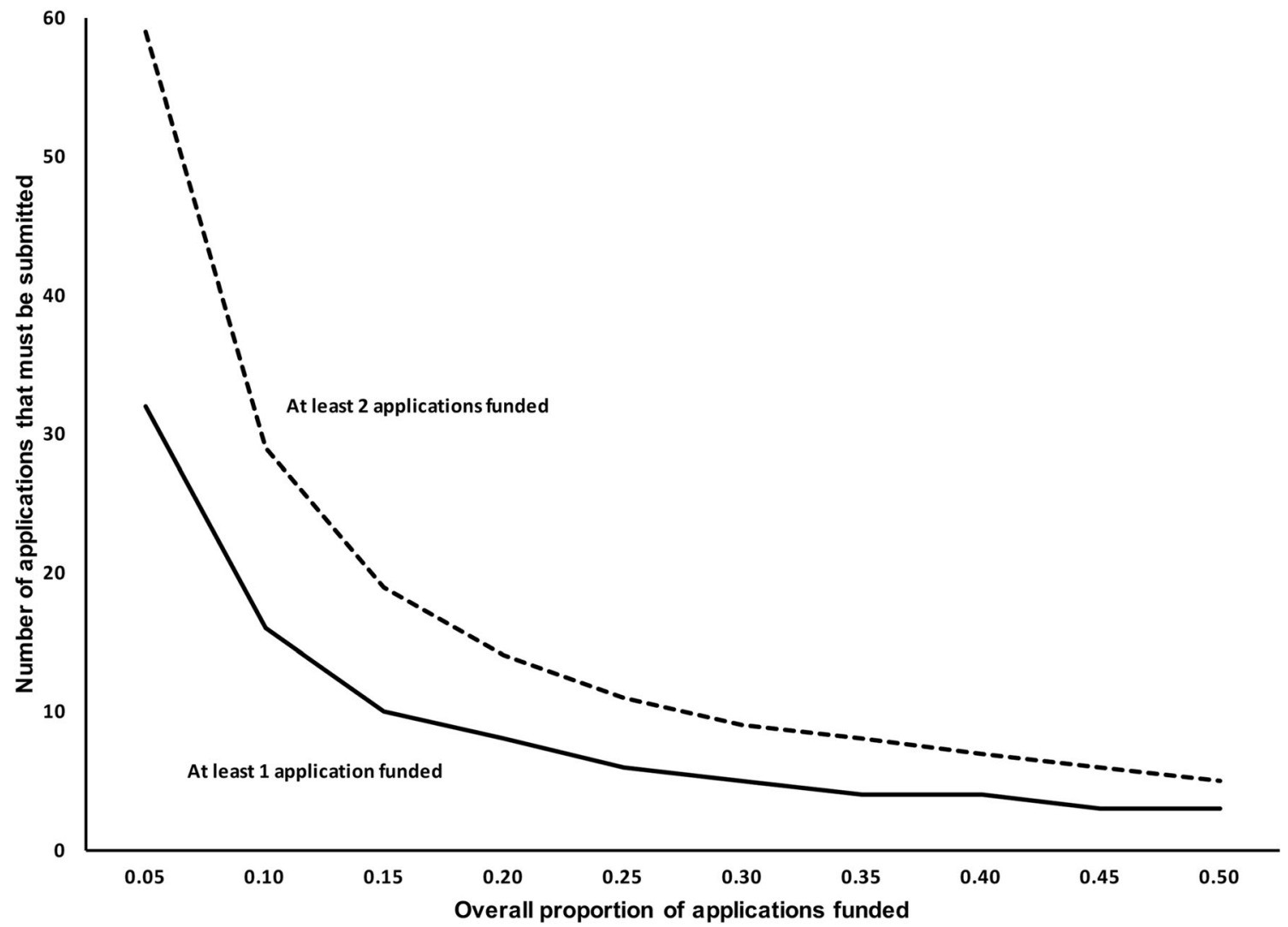

Figure 1 Number of grant applications that must be submitted to achieve $80 \%$ probability of having at least 1 or 2 applications funded.

addressed by assigning different funding rates to each application, e.g., an expected funding rate of 10 or $30 \%$ instead of the current NIH average funding rate of $20 \%$. In addition, the analyses are not based on actual results of grant submissions. However, it is likely that many investigators would give up before submitting 8-14 applications, so these probabilistic analyses are a good first step to understand this topic.

Corresponding Author: Ashwini R. Sehgal, MD; Center for Reducing Health Disparities, Division of Nephrology, Departments of Internal Medicine, Biomedical Ethics, and Epidemiology and Biostatistics, Metro Health Medical Center Case Western Reserve University, Cleveland, OH, USA (e-mail: sehgal@case.edu).
Funding information This paper is supported in part by grants MDO02265 and TR000439 from the National Institutes of Health.

\section{Compliance with Ethical Standards:}

Conflict of Interest: The author declares that he does not have a conflict of interest.

\section{REFERENCES}

1. Chin MH, Covinsky KE, McDermott MM, Thomas EJ. Building a research career in general internal medicine: a perspective from young investigators. J Gen Intern Med. 1998;13:117-22.

2. NIH Data Book. Available at https://report.nih.gov/nihdatabook/ Accessed 12 Mar 2018. 
3. Laudel G. The art of getting funded: how scientists adapt to their funding conditions. Sci Publ Policy. 2006;33:489-504.

4. Kulage KM, Schnall R, Hickey KT, et al. Time and costs of preparing and submitting an NIH grant application at a school of nursing. Nurs Outlook 2015;63:639-49.
5. Alberts B, Kirschner MW, Tilghman S, Varmus H. Rescuing US biomedical research from its systemic flaws. Proc Natl Acad Sci U S A. 2014;111:5773-7. 